\title{
molecules
}

ISSN 1420-3049

(C) 2007 by MDPI

www.mdpi.org/molecules

\author{
Full Paper
}

\section{New “Green” Approaches to the Synthesis of Pyrazole Derivatives}

\author{
Anna Corradi ${ }^{1}$, Cristina Leonelli ${ }^{1}$, Antonino Rizzuti ${ }^{1}$, Roberto Rosa ${ }^{1, *}$, Paolo Veronesi ${ }^{1}$, \\ Romano Grandi ${ }^{2}$, Sara Baldassari ${ }^{3}$ and Carla Villa ${ }^{3}$ \\ ${ }^{1}$ Dipartimento di Ingegneria dei Materiali e dell'Ambiente, Università degli Studi di Modena e \\ Reggio Emilia, Via Vignolese, 905, 41100 Modena, Italy. FAX: +390592056243; \\ tel: +390592056247; e-mails: corradi.anna@unimore.it, leonelli@unimore.it, \\ rizzuti.antonino@unimore.it, paolov@unimore.it
}

2 Dipartimento di Chimica, Università degli Studi di Modena e Reggio Emilia, Via Campi, 183, 41100 Modena, Italy; e-mail: romano@unimore.it

3 Dipartimento di Scienze Farmaceutiche dell'Università degli Studi di Genova, Viale Benedetto XV, 3, 16132 Genova, Italy; e-mails: s.baldassari@unige.it, carlavilla@unige.it

* Author to whom correspondence should be addressed; e-mail: rosa.roberto@unimore.it

Received: 31 May 2007; in revised form: 16 July 2007 / Accepted: 16 July 2007 / Published: 19 July 2007

\begin{abstract}
A novel approach to the synthesis of pyrazole derivatives from tosylhydrazones of $\alpha, \beta$-unsaturated carbonyl compounds possessing a $\beta$-hydrogen is proposed, exploiting microwave (MW) activation coupled with solvent free reaction conditions. The cycloaddition was studied on three ketones (trans-4-phenyl-3-buten-2-one, $\beta$-ionone and trans-chalcone). The corresponding 3,5-disubstitued-1H-pyrazoles were obtained in high yields and after short reaction times. In order to simplify and point out the green chemistry features of the method, a further improvement was achieved under the same catalytic conditions with a "one pot" synthesis of these heterocyclic compounds, starting directly from their carbonyl precursors via tosylhydrazones generated in situ. For an exhaustive study, the dielectric properties of the solid reaction mixtures were also measured, in order to obtain input data for the numerical simulation of their heating behaviour in the single mode MW cavity which was used for experimental work. In order to supply a valid methodology and tool for measuring the environmental impact, a comparative study between the synthetic route proposed and the classical synthetic route has been carried out.
\end{abstract}


Keywords: Pyrazole derivatives, solvent free cycloaddition, microwaves.

\section{Introduction}

Microwave (MW) irradiation has been widely exploited in the last decades to carry out a striking number of organic syntheses, benefiting from the dielectric heating in terms of reduced reaction times and increased yields, especially when coupled with solvent-free techniques. Generally three types of solvent-free procedures can be coupled with dielectric heating provided by a microwave source: reactions among neat reagents, reactions among supported reagents on mineral solid supports and phase transfer catalysis reactions. The neat reagents procedure is doubtlessly the most pursued one due, above all, to its easy work-up and negligible use of solvents for purification/separation stages. One of the most fertile applications in this field, also known as Microwave Assisted Organic Syntheses (MAOS) technique, is heterocyclic chemistry, as reported in a recent review [1]. In particular, the synthetic pathways of pyrazole derivatives represent an interesting topic since these compounds have numerous applications in the pharmaceutical and agrochemical industry [2].

Some of the most widespread synthetic strategies to obtain these heterocyclic structures are the reaction between hydrazines with $\beta$-difunctional compounds [3] and 1,3-dipolar cycloaddition of diazo compounds onto multiple bonds [4]. This last approach, which requires the preparation and the handling of the toxic and potentially explosive diazo compounds, is of secondary importance. Aggarwal et al. have reported [5] a method that represents a safe alternative for handling diazo compounds. This approach is based on the Bamford-Stevens reaction and uses tosylhydrazone salts as diazo precursors. They found that tosylhydrazone salts can be cleanly converted to diazo compounds under mild reaction conditions and in a wide range of solvents. These diazo compounds can then be induced to react directly with alkenes or alkynes to synthesize pyrazoles after additional steps.

Bamford and Stevens first reported in 1952 the base mediated decomposition of substituted arylhydrazones to form diazo compounds [6]. A few diazo compounds could be isolated if mild temperatures were employed, but in most cases, the diazo compounds decomposed thermally to form alkenes as a result of loss of dinitrogen followed by a 1,2-hydrogen shift. Another synthetic route towards pyrazole derivatives, first demosntrated by Hart and Brewbaker, consists of an intramolecular cycloaddition of 3-diazoalkenes generated from the corresponding ethyl alkenylnitrosocarbamates [7]. Doyle et al. [8] observed formation of pyrazoles during reaction of the corresponding tosylhydrazone salt with $\mathrm{Rh}_{2}(\mathrm{OAc})_{4}$. A one-pot synthesis of 3(5)-substituted-1H-pyrazoles from aldehydes and diethoxyphosphorylacetaldehyde tosylhydrazone is also described in the literature [9]. Another one-pot approach has been proposed by Aggarwal et al., using diazo compounds generated in situ from tosylhydrazone salts [2]. They studied two different routes to pyrazoles: first, direct 1,3-cycloaddition of diazo compounds onto alkynes, and second, employing an olefin bearing a leaving group, which would afford the pyrazole after an elimination/aromatization of the cycloadduct intermediate. Moreover Grandi et al. [10] demonstrated that tosylhydrazones of some acyclic $\alpha, \beta$-unsaturated carbonyl compounds containing an hydrogen atom in $\beta$ position, on treatment with $\mathrm{NaBH}_{4}$ or $\mathrm{CH}_{3} \mathrm{ONa}$ or $\mathrm{K}_{2} \mathrm{CO}_{3}$ in alcoholic solvents (such as $\mathrm{CH}_{3} \mathrm{OH}$ ) can lead to an intramolecular 1,3-dipolar cycloaddition to give pyrazole derivatives (Scheme 1 ). 
Scheme 1. Reaction mechanism for intramolecular 1,3-dipolar cycloaddition of tosylhydrazones derived from acyclic $\alpha, \beta$-unsaturated carbonyl compounds with a $\beta-$ hydrogen.

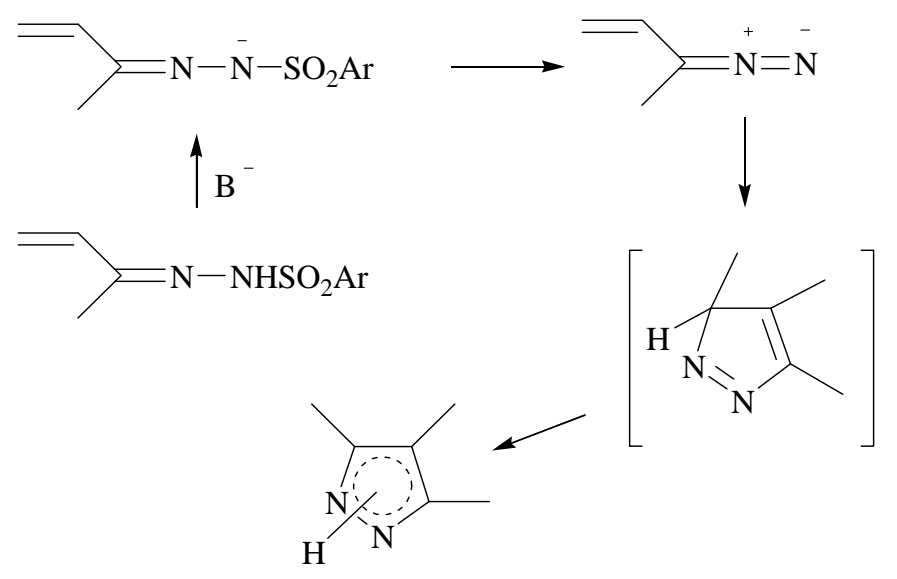

\section{Results and Discussion}

Synthesis

On the basis of these studies in the present work we report simple and mild solvent-free microwave mediated methodologies for the synthesis of pyrazoles derivatives from $\alpha, \beta$-unsaturated carbonyl compounds.

Table 1. Physical and chemical properties of products a-c obtained under microwave activation.

\begin{tabular}{|c|c|c|c|c|c|}
\hline Compound & $\mathbf{R}$ & $\mathbf{R}^{\prime}$ & $\mathbf{m p},{ }^{\circ} \mathrm{C}^{a}$ & $\begin{array}{l}\text { bp, }{ }^{\circ} \mathrm{C}^{b} \\
\text { (mmHg) }\end{array}$ & $\begin{array}{c}{ }^{1} \text { H-NMR data, } \\
\delta / \text { ppm }\end{array}$ \\
\hline $\mathbf{a}$ & $\mathrm{C}_{6} \mathrm{H}_{5}$ & $\mathrm{CH}_{3}$ & $124-126^{*}$ & - & $\begin{array}{c}2.31\left(3 \mathrm{H}, \mathrm{s}, \mathrm{CH}_{3} \mathrm{C}(3)\right), \\
6.35(1 \mathrm{H}, \mathrm{s}, \mathrm{C}(4) H), \\
7.30-7.75(5 \mathrm{H}, \text { aromatic })\end{array}$ \\
\hline b & & $\mathrm{CH}_{3}$ & - & $\begin{array}{c}165-170 \\
(0.1)\end{array}$ & $\begin{array}{c}0.95\left(6 \mathrm{H}, \mathrm{s},\left(\mathrm{CH}_{3}\right)_{2} \mathrm{C}\right) \\
1.42\left(3 \mathrm{H}, \mathrm{s}, \mathrm{CH}_{3} \mathrm{C}=\mathrm{C}\right) \\
2.31\left(3 \mathrm{H}, \mathrm{s}, \mathrm{CH}_{3} \mathrm{C}(3)\right) \\
5.80(1 \mathrm{H}, \mathrm{s}, \mathrm{C}(4) \mathrm{H})\end{array}$ \\
\hline c & $\mathrm{C}_{6} \mathrm{H}_{5}$ & $\mathrm{C}_{6} \mathrm{H}_{5}$ & $200-202^{* *}$ & - & $\begin{array}{c}6.85(1 \mathrm{H}, \mathrm{s}, \mathrm{C}(4) \mathrm{H}), \\
7.32-7.74(10 \mathrm{H}, \\
\text { aromatic })\end{array}$ \\
\hline
\end{tabular}

${ }^{*}$ Crystallization solvent $=96 \%$ ethanol $;{ }^{* *}$ crystallization solvent $=$ cyclohexane.

${ }^{a} \mathrm{Mp}$ reported in the literature for compound a: $125-126^{\circ} \mathrm{C}$ [10]; for compound c: $200{ }^{\circ} \mathrm{C}[11]$.

${ }^{\boldsymbol{b}} \mathrm{Bp}$ reported in the literature for compound $\mathbf{b}$ : $148-150{ }^{\circ} \mathrm{C}$ (0.01 $\left.\mathrm{mmHg}\right)[10]$. 
We investigated in detail three carbonyl substrates: trans-4-phenyl-3-buten-2-one, $\beta$-ionone and trans-chalcone. Compounds a-c (Table 1) have been obtained under microwave activation following two methodologies (MW1 and MW2).

The first approach to synthesize the pyrazole derivatives (MW1) refers to the 1,3-cycloaddition of the corresponding tosylhydrazones of the carbonyl compounds selected, using a base in dry media (Scheme 2). Tosylhydrazones were prepared following the classical procedure (ketone plus tosylhydrazine in methanol at reflux) [12].

Scheme 2. Reaction scheme for the synthesis of pyrazoles a-c from tosylhydrazones.

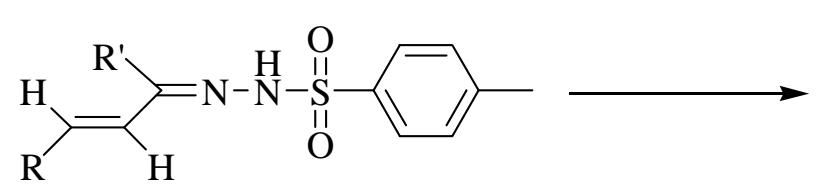<smiles>[R]c1n[nH]nc1[R]</smiles>

a-c

To fine-tune the method, and using the synthesis of compound a from the tosylhydrazone of trans4-phenyl-3-buten-2-one as a model reaction, several experiments were carried out under microwave activation using a base impregnated on mineral solid supports, a neat base in the reaction mixture, a base with an energy transfer agent such as $N, N$-dimethylformamide or graphite. The most remarkable results are reported in Table 2.

Table 2. Operative conditions and relative yields for the synthesis of product a from tosylhydrazone.<smiles>Cc1cc(-c2ccccc2)n[nH]1</smiles>

a

\begin{tabular}{ccccc}
\hline Entry & Method & $\begin{array}{c}\text { Time, } \\
\text { min. }\end{array}$ & $\begin{array}{c}\text { Temp., } \\
{ }^{\circ} \mathbf{C}\end{array}$ & Yield, \% \\
\hline A & $\mathrm{K}_{2} \mathrm{CO}_{3}(8: 1)^{*}$ & 45 & 150 & n.r. \\
\hline B & $\mathrm{K}_{2} \mathrm{CO}_{3} / \mathrm{KOH}(1 / 1: 1)^{*}$ & 45 & 145 & 84 \\
\hline C & $\mathrm{KF} \mathrm{Al}_{2} \mathrm{O}_{3}(1 / 1: 1)^{*}$ & 10 & 145 & $65^{* *}$ \\
\hline D & $\mathrm{K}_{2} \mathrm{CO}_{3}(4: 1)^{*}+\mathrm{ETI} 1$ & 10 & 150 & $62^{* *}$ \\
\hline E & $\mathrm{K}_{2} \mathrm{CO}_{3}(4: 1)^{*}+\mathrm{ETI} 2$ & 1 & 130 & 95 \\
\hline F & $\mathrm{K}_{2} \mathrm{CO}_{3}(2: 1)^{*}+\mathrm{ETI} 2$ & 1 & 130 & 95 \\
\hline
\end{tabular}

* Molar ratio

ETI1 = Energy transfer improver: powdered graphite (catalytic amount)

ETI2 = Energy transfer improver: DMF (catalytic amount)

**Remainder $=$ degradation compounds 
In general, the simple mixing of neat reagents gave better results than mineral solid supports in terms of reaction times and yields. Only the use of $\mathrm{K}_{2} \mathrm{CO}_{3}$ as a base, without any energy transfer improver, did not lead to any product. The addition of an energy transfer improver enhanced the reaction speed and yields and allowed us to reduce both the reaction temperature and amount of the base. The best performing conditions resulted from neat reagents in the presence of anhydrous $\mathrm{K}_{2} \mathrm{CO}_{3}$ (substrate/base 1:2) and a catalytic amount of $N, N$-dimethylformamide (DMF) as an energy transfer improver [13]. Figure 1 shows the temperature and power profiles corresponding to entry F in Table 2 for the MW1 synthetic protocol of product a. This method was then applied to the synthesis of all compounds. The results are reported in Table 3.

Figure 1. Graph of temperature and power \% vs time for the synthesis of pyrazole a following MW1 synthetic approach under the entry F (Table 2) operative conditions.

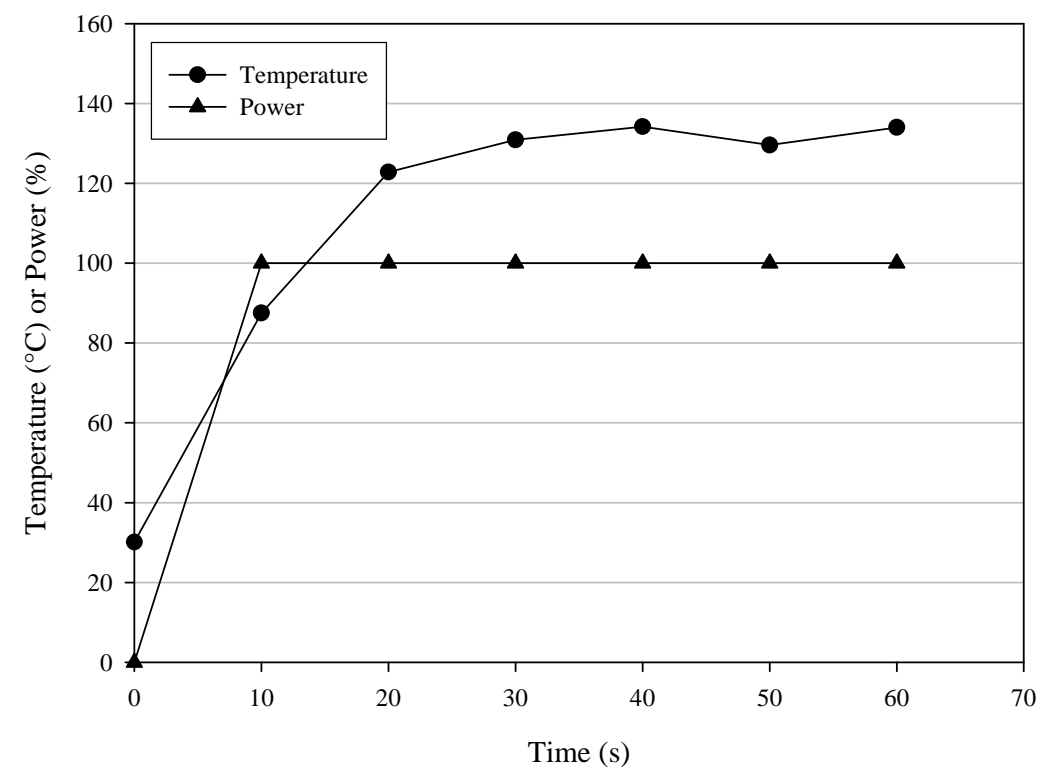

In order to evaluate how microwave irradiation can affect cyclization, the best performing method (entry F) was also carried out under conventional heating (thermostated oil bath) under the same conditions in terms of reagents quantities and reaction vessel, in order to obtain compound a. Moreover, due to the slower temperature increase of conventional heating, reaction time was considered starting from when the temperature setpoint $\left(130^{\circ} \mathrm{C}\right)$ was reached. No product was detected after one minute of conventional treatment at $130{ }^{\circ} \mathrm{C}$. When conventional heating was extended TLC monitoring of the oil bath reaction mixture revealed that the substrate started reacting only after 10 minutes, but then some degradation compounds were also detected.

In the second phase of the work (MW2), as a further improvement and simplification, a "one-pot" approach to the synthesis of the desired heterocyclic products was investigated, removing the tosylhydrazone formation step. Hence, starting directly from the carbonyl precursors (Scheme 3) and using the catalytic conditions which gave the best results in the previous approach (entry $\mathbf{F}=$ $\mathrm{K}_{2} \mathrm{CO}_{3}$ /substrate 2:1, $130{ }^{\circ} \mathrm{C}$, DMF as energy transfer improver), the synthesis of the pyrazole derivatives a-c was studied at different reaction times, monitoring the product formation by TLC. The results obtained from this last synthetic pathway are reported in Table 4. As an example, Figure 2 shows the temperature and power profiles for MW2 synthetic protocol of product a. 
Table 3. Solvent free microwave-mediated synthesis (MW1) of compounds a-c under the best catalytic conditions (entry $\mathbf{F}$ - Table 2) from tosylhydrazones.

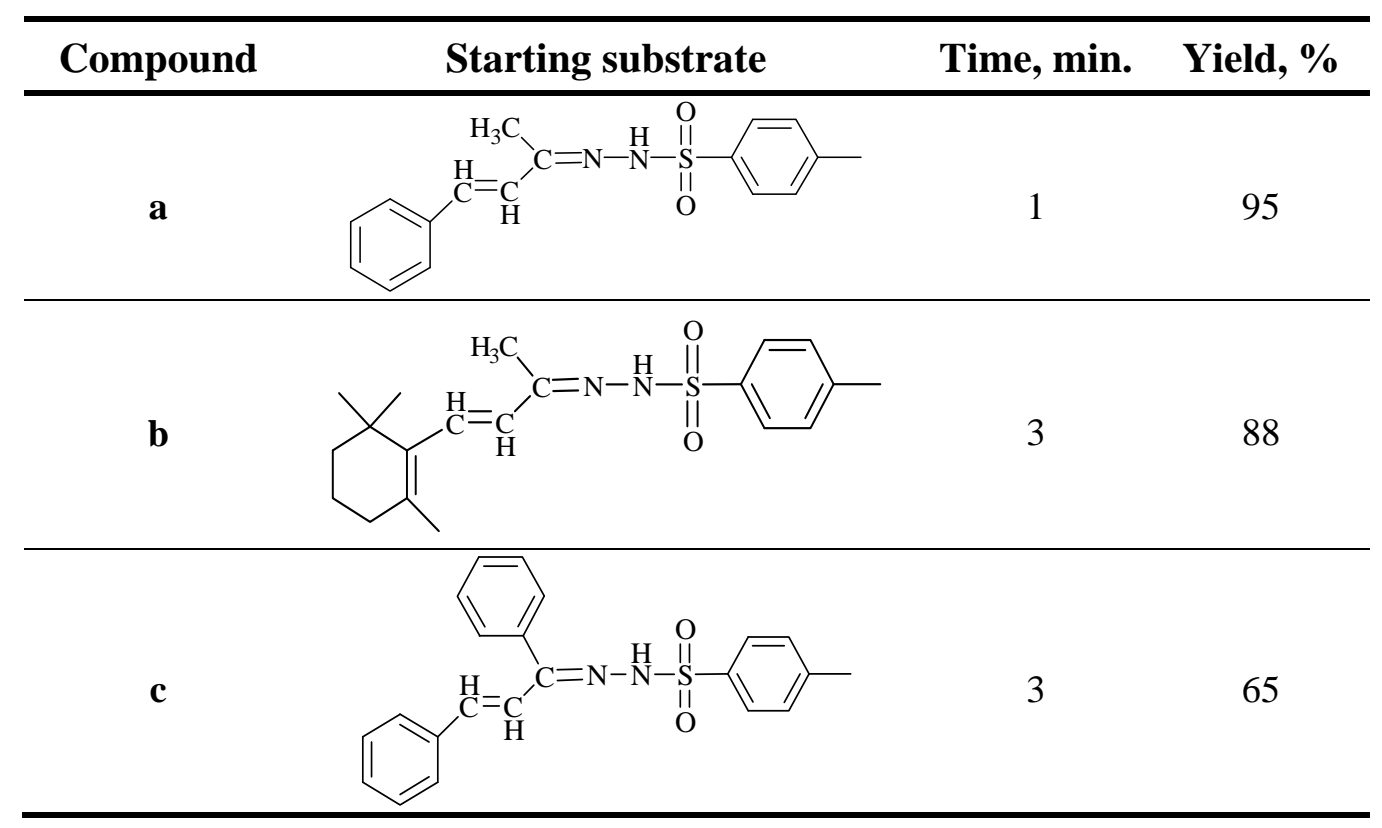

Scheme 3. Reaction scheme for the synthesis of pyrazoles a-c starting directly from carbonyl precursors.<smiles>[R]C=CC([R])=O</smiles><smiles></smiles>

a-c

Table 4. MW-assisted “one pot synthesis” (MW2) of compounds a-c from carbonyl precursors.

\begin{tabular}{llll}
\hline Product & Tharting substrate & $\begin{array}{c}\text { Time, } \\
\text { min. }\end{array}$ & $\begin{array}{c}\text { Yield, } \\
\%\end{array}$ \\
\hline a & 3 & \\
\hline
\end{tabular}


Figure 2. Graph of temperature and power \% vs time for the synthesis of pyrazole a following MW2 synthetic approach under the entry F (Table 2) operative conditions.

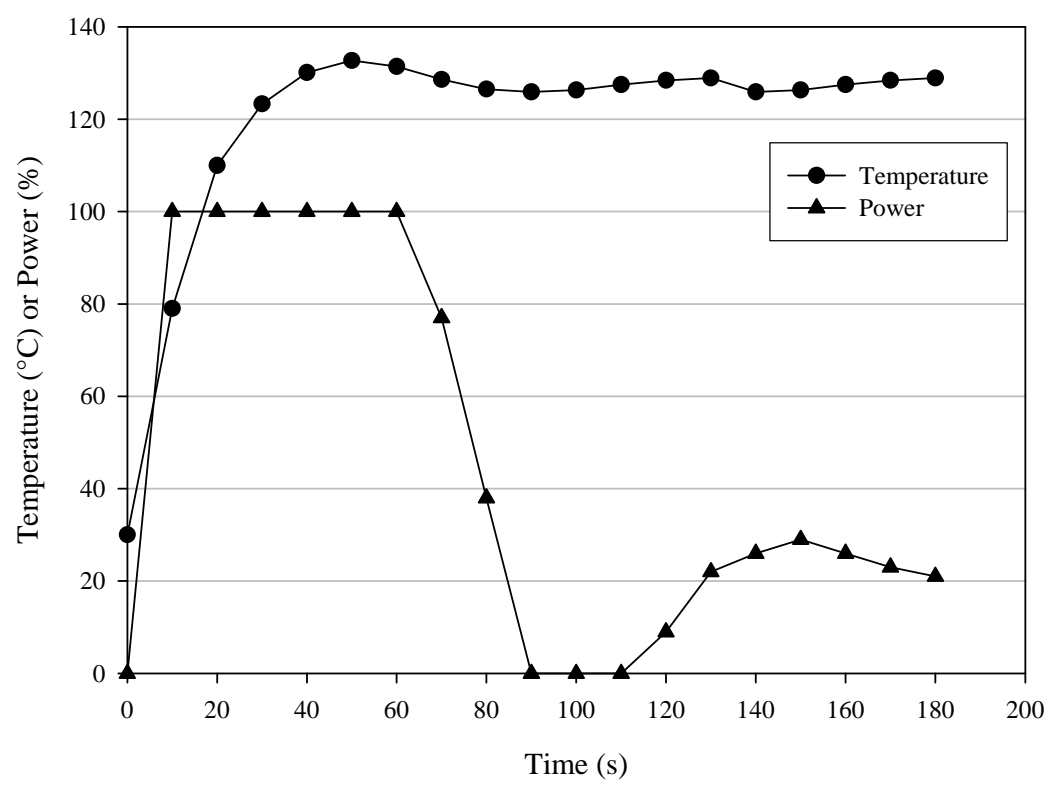

With a simple mixing of the reagents (ketone, tosylhydrazide, $\mathrm{K}_{2} \mathrm{CO}_{3}$ and DMF) and with a maximum reaction time of five minutes all the compounds were obtained in good yields, very close to those obtained using the best catalytic conditions of MW1 (Yield \% entry F: MW1/one pot: compound $\mathbf{a}=95 / 95$, compound $\mathbf{b}=88 / 84$, compound $\mathbf{c}=65 / 65$ ).

Again, in order to evaluate how microwave irradiation affected the cyclization, the "one pot" synthesis of compound a was also carried out under conventional heating (thermostated oil bath) under the same conditions in terms of reagents quantities and reaction vessel used. No product was detected after 3 minutes of treatment at $130{ }^{\circ} \mathrm{C}$. The reaction in oil bath was also monitored for longer reaction times and after 10 minutes of treatment at $130{ }^{\circ} \mathrm{C}$ some degradation products were detected.

\section{Modelling}

The software Concerto 4.0 (Vector Fields, U.K.) has been used to numerically simulate the heating behaviour of the reactants mixture in the MW single mode applicator. The numerical simulation helped explaining the different yields and cleanness measured when using different reactants in the same experimental conditions.

The study of microwave heating of the reactants was conducted only for the MW-assisted one pot synthesis of products a-c (Table 3). The use of the Concerto software allowed us to determine the SAR (Specific Absorption Rate, in $\mathrm{W} / \mathrm{kg}$ ), and thus to describe how heat is generated within the reaction vessel. The calculated SAR in the mid horizontal section of the reaction vessel is reported in the thermal plots shown in Figure 3, as a function of compounds a-c. 
Figure 3. SAR in the mid section of the reaction vessel centrally positioned in the single mode applicator (300W forward power).

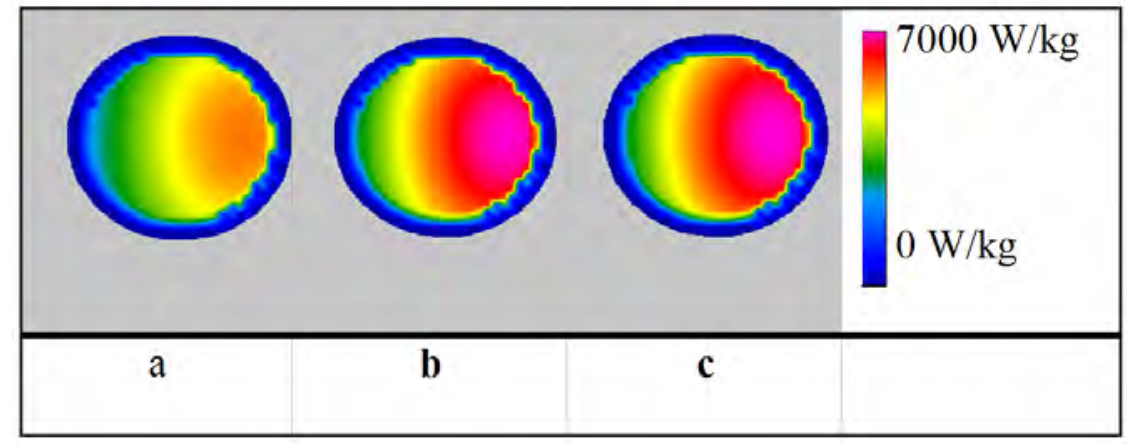

The SAR distribution shows non-homogenous heating in the case of stationary load (power generation higher in the right side of the load), which, however, can be compensated by stirring. This distribution is due to the presence of the load inside the cavity, which is not equipped with plungers or movable short circuits to alter the electric field distribution. As a matter of fact, the load perturbs the empty cavity electric field distribution, having the maximum intensity region move away from the centre of the sample. This unwanted effect, on the other side, results useful in case of sample stirring (rotation), since it allows a much higher homogeneity of power generation with respect to a configuration in which the maximum of the electric field would be in the exact centre of the load. Modeling results show also that the solid reaction mixture for product a synthesis presents a $30 \%$ lower power generation in the examined section. However, it was experimentally observed that this particular sample (a) is the one that lead to the cleanest products and higher yield reaction. This behaviour could be ascribed to the easier and more accurate temperature control of the reaction in the case of lower power generation by the reactants (temperature gradients are reduced). On the other hand, in this case the overall process energy efficiency is lower, suggesting that an improved stirring and homogenisation could lead to higher yields for products $\mathbf{b}$ and $\mathbf{c}$ as well provided the synthesis conditions can be effectively controlled, minimising localised over- or under- heating.

\section{Green metrics evaluation}

A comparative quantitative assessment of the "greenness" of the new processes here proposed, in comparison to the classical one [10], was conducted with a rational approach of the chemical sustainability of a reaction ("Green metrics”). For this the "Environmental Assessment Tool for Organic Syntheses” software (EATOS) was used [14, 15]. This software, which takes into account the mass economy as well as the environmental and human health impact of the materials involved, has been applied to assess the eco-sustainability of the competing processes. EATOS elaborates all data relative to all materials used in the process (reagents, solvents, auxiliary materials, products and secondary products) and supplies an evaluation histogram. It is also possible to compare two or more chemical processes placing side by side their histograms and standardizing the quantities. As an example, in Figure 4 the histograms related to the environmental impact of the synthesis of compound a using both the conventional route in methanol [10], and the newly proposed microwave approaches 
are reported. As the order of magnitude of the environmental impact of the classic procedure is much higher, the green metrics relative to MW1 and MW2 processes are also shown in detail in Figure 5.

Figure 4. Evaluation histograms of classic versus MW-mediated solvent-free approaches, MW1 and MW2 (one pot), for the synthesis of compound a. $\mathrm{S}^{-1}=$ inverse selectivity (or mass index). $\mathrm{E}=$ environmental factor. $\mathrm{EI}_{\text {in }}$ and $\mathrm{EI}_{\text {out }}$ represent respectively $\mathrm{S}^{-1}$ and $\mathrm{E}$ weighted in function of the "weighting factors": costs, risk, human toxicity, ecotoxicology. PEI represents Possible Environmental Impact.

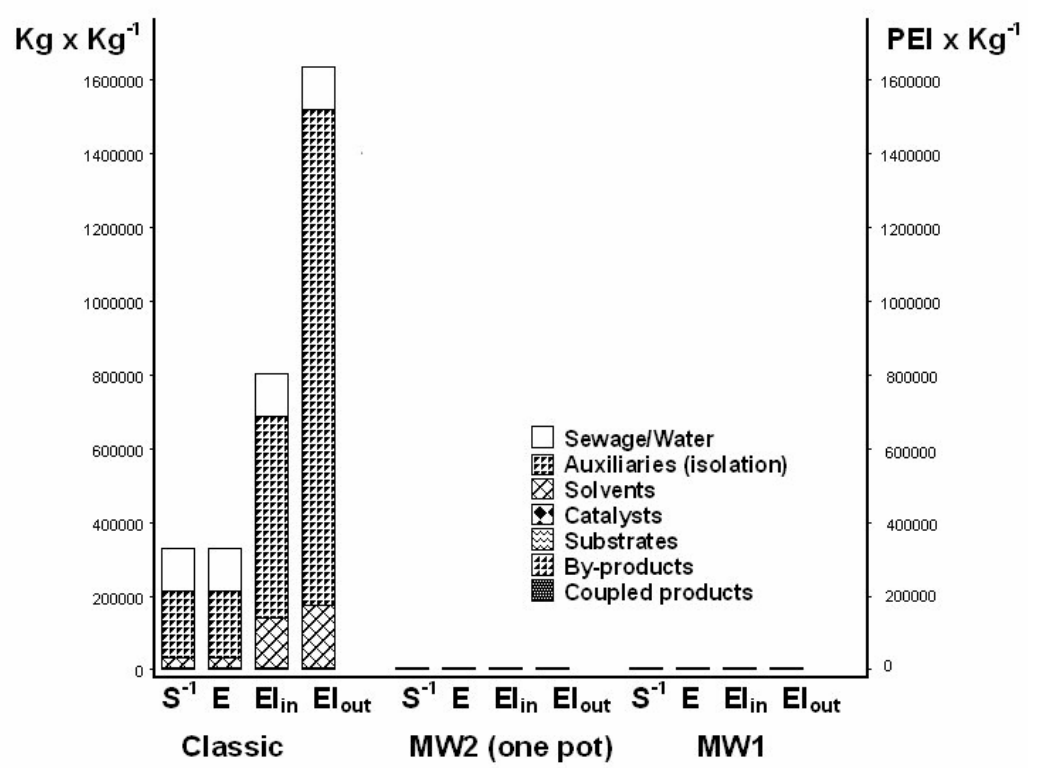

Figure 5. Evaluation histograms of MW mediated solvent-free approaches, MW1 and MW2 (one pot), for the synthesis of compound a. $\mathrm{S}^{-1}=$ inverse selectivity (or mass index). $\mathrm{E}=$ environmental factor. EIin and EIout represent respectively $\mathrm{S}^{-1}$ and $\mathrm{E}$ weighted in function of the "weighting factors" (costs, risk, human toxicity, ecotoxicology). PEI represents Possible Environmental Impact.

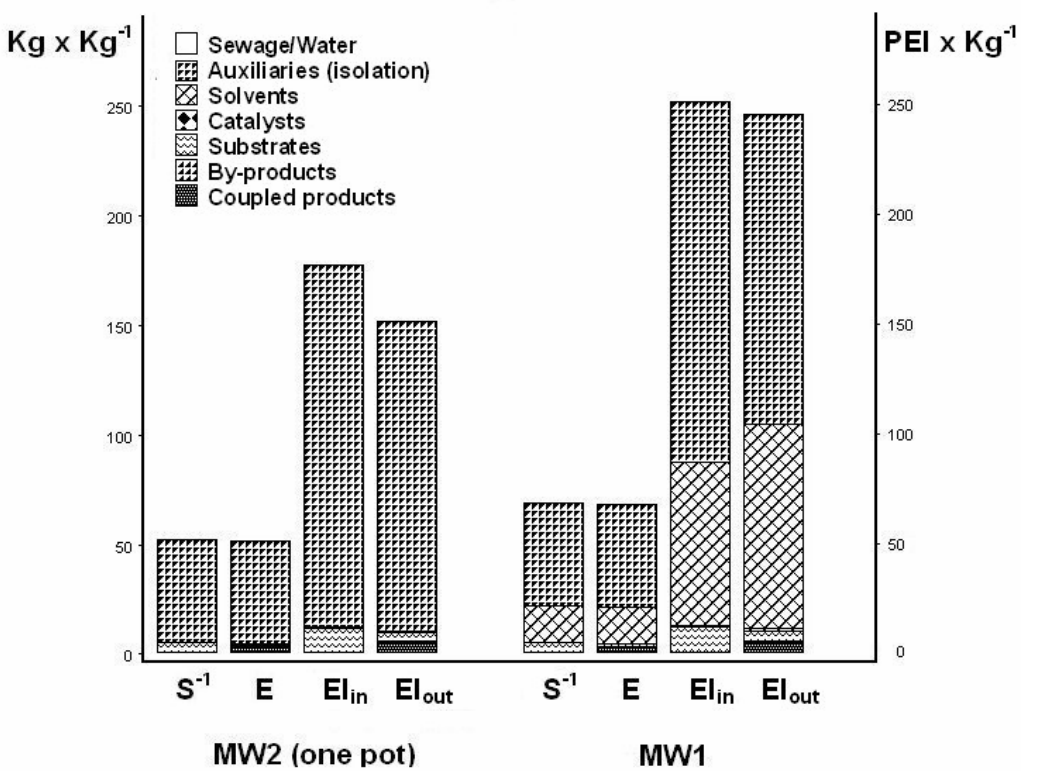


The graphical results shown in Figure 4 suggest that the performance indicators of our innovative methods are considerably improved from a green chemistry point of view. A further confirmation can be given by the rapidity of the process which is not considered by EATOS but which is important in a green chemistry procedure context in terms of reduction of energy consumption.

\section{Conclusions}

The good results obtained in terms of reaction speed and yields with the proposed microwavemediated solvent-free processes indicate that these procedures can be considered simple, fast and ecofriendly synthetic pathways to obtain pyrazole derivatives from $\alpha, \beta$-unsaturated ketones with a $\beta$ hydrogen. This is confirmed by the EATOS software, especially with regards to the new "one-pot" method. On equal terms of yield and quite similar reaction times, this last approach has to be considered the best synthetic route taking into account atom economy, step reduction and further methanol elimination in the synthesis of tosylhydrazones, in full agreement with the Green Chemistry principles.

\section{Experimental}

\section{General}

trans-4-Phenyl-3-buten-2-one ( $\geq 99 \%)$, $\beta$-ionone (96\%), trans-chalcone (97\%), $p$-toluenesulfonylhydrazide ( 99\%), $\mathrm{K}_{2} \mathrm{CO}_{3}, \mathrm{KF}, \mathrm{Al}_{2} \mathrm{O}_{3}, \mathrm{KOH}$ and graphite flakes were provided by Sigma-Aldrich (Milan, Italy). $N, N$-dimethylformamide and diethyl ether were provided by Merck (Darmstadt, Germany). MW irradiation was supplied, for all samples, by a commercial Synthewave ${ }^{\mathrm{TM}} 402-$ Prolabo monomode reactor specifically designed for organic synthesis. This reactor operates at 2.45 $\mathrm{GHz}$ of frequency with a maximum output power of $300 \mathrm{~W}$. The temperature measurement is supplied by an IR thermometer pointed to the external wall of the reaction vessel. The instrument's software is able to continuously varying the output power as a function of the temperature detected. A more rigorous measurement of the load local temperature was obtained using one or more optical fibers (Neoptix Reflex, 4 channels) introduced into the bulk reaction. Homogenous mechanical stirring of the solid samples was obtained thanks to a curved Pyrex rod fixed on the top of the cavity while the reaction vessel continuously rotates at $60 \mathrm{rpm}$. IR spectra were acquired with a Bruker Vertex 70 FTIR spectrometer provided with an ATR cell. ${ }^{1} \mathrm{H}-\mathrm{NMR}$ spectra were recorded on a Bruker Advance DPX instrument (400 MHz) with TMS as an internal standard; chemical shifts are reported as $\delta$ (ppm) relative to TMS. Elemental analyses were performed on CE Instruments - EA 1110 CHNS-O. Melting and boiling points were determined on a Büchi 510 apparatus and are uncorrected. Oil bath temperature measurements were carried out using a Comark digital thermometer.

\section{Microwave mediated synthesis of pyrazoles a-c (MW1). General procedure}

In a $40 \mathrm{~mL}$ Pyrex tube, the appropriate tosylhydrazone of the studied carbonyl compounds (10 mmol), previously prepared by a classical procedure [12], was mixed together with anhydrous $\mathrm{K}_{2} \mathrm{CO}_{3}$ 
(20 mmol). A minimum amount of $N, N$-dimethylformamide (30 $\mathrm{mg} / \mathrm{mmol}$ of organic substrate) was added in order to improve energy transfer [13]. The reaction mixture was placed in the microwave cavity and irradiated under stirring at $130{ }^{\circ} \mathrm{C}$ for an appropriate time (as reported in Table 2), maintaining this temperature by power modulation from 5 to $300 \mathrm{~W}$. The product formation was monitored during all the processes by TLC (silica gel plates with fluorescent indicator, diethyl ether/ petroleum ether 9:1 as mobile phase). After cooling to room temperature, the organic product was recovered by elution with diethyl ether $(50 \mathrm{~mL})$ and filtration on a Gooch filter. The solution was evaporated under reduced pressure and the crude product was purified by recrystallization with the appropriate solvent or by bulb-to-bulb distillation under vacuum.

Synthesis of pyrazole a from tosylhydrazone under conventional heating. General Procedure

In a $40 \mathrm{~mL}$ Pyrex tube, tosylhydrazone of trans-4-phenyl-3-buten-2-one (10 mmol), previously prepared by a classical procedure [12], was mixed together with anhydrous $\mathrm{K}_{2} \mathrm{CO}_{3}$ (20 mmol). A minimum amount of $N, N$-dimethylformamide (30 mg/mmol of organic substrate) was added (as in microwave procedure) [13]. The reaction mixture was heated under stirring by a thermostated oil bath settled at $130{ }^{\circ} \mathrm{C}$. Reaction time was considered when the reaction bulk reached this temperature. Product formation was monitored by TLC (silica gel plates with fluorescent indicator, diethyl ether/ petroleum ether 9:1 as mobile phase).

\section{Microwave-mediated “one-pot synthesis” of pyrazoles a-c (MW2). General procedure}

In a $40 \mathrm{~mL}$ Pyrex tube, the appropriate $\alpha, \beta$-unsaturated ketone $(10 \mathrm{mmol})$ was mixed in the reaction vessel with a stoichiometric amount of $p$-toluenesulfonhydrazide. After a gentle stirring 20 mmol of $\mathrm{K}_{2} \mathrm{CO}_{3}$ and a minimum amount of $N, N$-dimethylformamide (30 $\mathrm{mg} / \mathrm{mmol}$ of organic substrate) were added. The reaction mixture was placed in the microwave cavity and irradiated under stirring at $130{ }^{\circ} \mathrm{C}$ for the appropriate time (as reported in Table 3), maintaining this temperature by power modulation from 5 to $300 \mathrm{~W}$. The product formation was monitored by TLC in the same conditions of the MW1 method. After cooling to room temperature, the organic product was recovered as described in the previous procedure.

\section{“One pot” synthesis of compound a under conventional heating. General Procedure}

In a $40 \mathrm{~mL}$ Pyrex tube, $10 \mathrm{mmol}$ of trans-4-phenyl-3-buten-2-one was mixed in the reaction vessel with a stoichiometric amount of p-toluenesulfonhydrazide. After a gentle stirring $20 \mathrm{mmol}$ of $\mathrm{K}_{2} \mathrm{CO}_{3}$ and a minimum amount of $N, N$-dimethylformamide (30 $\mathrm{mg} / \mathrm{mmol}$ of organic substrate) were added. The reaction mixture was heated under stirring by a thermostated oil bath settled at $130{ }^{\circ} \mathrm{C}$. Reaction time was considered when the reaction bulk reached this temperature. Product formation was monitored by TLC (silica gel plates with fluorescent indicator, diethyl ether/ petroleum ether 9:1 as mobile phase). 


\section{Modelling}

During microwave irradiation, the presence of the reaction vessel within the monomode cavity deeply influences the heating behaviour and the energy efficiency of the whole process, modifying the electromagnetic field distribution. The model used to better evaluate the heating process was chosen so as to represent the inner metal structure of the Synthewave ${ }^{\mathrm{TM}} 402$ single mode applicator as shown in Figure 6.

Figure 6. Microwave cavity of the Prolabo Synthewave ${ }^{\mathrm{TM}} 402$.

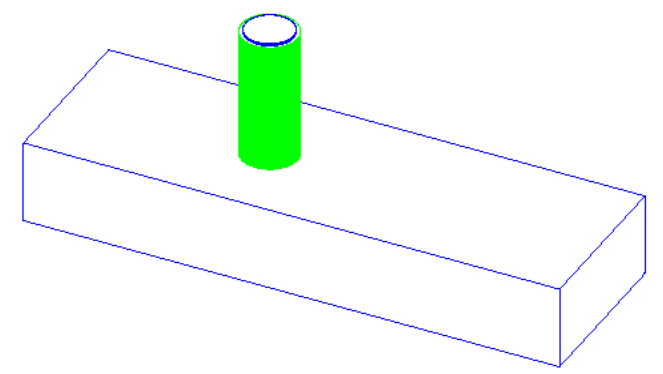

It is composed of a rectangular metallic enclosure (microwave applicator) containing in the central position a $24 \mathrm{~mm}$ diameter vessel made of Pyrex glass, thickness $2 \mathrm{~mm}$, filled up to $20 \mathrm{~mm}$ height with the load (the three different solid reaction mixtures for one pot syntheses of product a-c), whose dielectric properties were measured at room temperature in the 1-3 GHz frequency range by an Agilent 85070E Dielectric Probe Kit connected to a vector network analyser. On one side of the applicator is positioned a port excited sinusoidally in the fundamental TE10 mode, simulating the microwave generator (magnetron), operating at $300 \mathrm{~W}$ power, $2.45 \mathrm{GHz}$ frequency.

\section{Green metrics evaluation}

The EATOS software, which takes into account the mass economy as well as the environmental and human health impact of the materials involved, has been applied to assess the "greenness" of the competing processes. EATOS elaborates all data relative to all materials used in the process (reagents, solvents, auxiliary materials, products and secondary products) and supplies an evaluation histogram. It is also possible to compare two or more chemical processes placing side by side their histograms and standardizing the quantities. In such histograms, the first column is the inverse selectivity $\mathrm{S}^{-1}$ (or mass index). $\mathrm{S}^{-1}$ is the weight ratio between the sum of all input materials and the final products (Equation 1). The environmental factor $E$ in the second column is the weight ratio between the waste and the final products (Equation 2). In the third column, EI_in represents $\mathrm{S}^{-1}$ weighted in function of the weighting factors while column fourth is $\mathrm{E}$ weighted in function of the weighting factors. The weighting factors considered for input materials were price (significance of 50\%) and risk expressed by the R-phrases (significance of 50\%). On the other hand, the weighting factors considered for output materials were toxicity on human being, chronic- and eco- toxicity, each with a significance of 33\%. 
Equation 1. Mathematical expression of the mass index $\mathrm{S}^{-1}$

mass index $\mathrm{S}^{-1}=\frac{\sum \text { substrate }[\mathrm{g}]+\text { solvent }[\mathrm{g}]+\text { auxiliary material }[\mathrm{g}]+\text { cat. }[\mathrm{g}]+\ldots}{\text { product }[\mathrm{g}]}$

Equation 2. Mathematical expression of the environmental factor $E$

$$
\text { environmental factor } \mathrm{E}=\frac{\sum \text { waste }[\mathrm{g}]}{\text { product }[\mathrm{g}]}
$$

\section{References and Notes}

1. Bougrin, K.; Loupy, A. Microwave-Assisted Solvent-Free Heterocyclic Synthesis. J. Photochem. Photobiol. C, Photochem. Rev. 2005, 6, 139-167.

2. Aggarwal, V. K.; de Vicente, J.; Bonnert R. V. A Novel One-Pot Method for the Preparation of Pyrazoles by 1,3-Dipolar Cycloadditions of Diazo Compounds Generated in Situ. J. Org. Chem. 2003, 68, 5381-5383.

3. Kost, A. N.; Grandberg, I. I. Progress in Pyrazole Chemistry. Adv. Heterocycl. Chem. 1966, 6, 347-429.

4. Padwa, A. 1,3-Dipolar Cycloaddition Chemistry; John Wiley \& Sons: New York, 1984; Vol.I.

5. Fulton, J. R.; Aggarwal, V. K.; de Vicente, J. The Use of Tosylhydrazone Salts as a Safe Alternative for Handling Diazo Compounds and Their Applications in Organic Synthesis. Eur. J. Org. Chem. 2005, 1479-1492.

6. Bamford, W. R.; Stevens, T. S. The Decomposition of p-Tolylsulfonylhydrazones by Alkali. $J$. Chem. Soc. 1952, 4735-4740.

7. Brewbaker, J. L.; Hart, H. The Cyclization of 3-Diazoalkenes to Pyrazoles. J. Am. Chem. Soc. 1969, 91, 711-715.

8. Doyle, M. P.; Yan, M. J. Effective and Highly Stereoselective Coupling with Vinyldiazomethanes to Form Symmetrical Trienes. J. Org. Chem. 2002, 67, 602-604.

9. Almirante, N.; Cerri, A.; Fedrizzi, G.; Marazzi, G.; Santagostino, M. A General, [1+4] Approach to the Synthesis of 3(5)-Substituted Pyrazoles from Aldehydes. Tetrahedron Lett. 1998, 39, 32873290.

10. Grandi, R.; Messerotti, W.; Pagnoni, U. M.; Trave, R. Decomposition of Coniugated pTosylhydrazones in Base. Partition between Solvolysis and Cycloaddition Products. J. Org. Chem. 1977, 42, 1352-1355.

11. Bhat, B. A.; Puri, S. C.; Qurishi, M. A.; Dhar, K. L.; Qazi, G. N. Synthesis of 3,5-Diphenyl-1HPyrazoles. Synth. Comm. 2005, 35, 1135-1142.

12. Grandi, R.; Marchesini, A.; Pagnoni, U. M.; Trave, R. Conversion of Coniugated pTosylhydrazones to the Corresponding Ethers by Sodium Borohydride, Sodium Alkoxide, or Potassium Carbonate in Alcohol Solvents. J. Org. Chem. 1976, 41, 1755-1758. 
13. Rodriguez, H.; Perez, R.; Suarez, M.; Lam, A.; Cabrales, N.; Loupy, A. Alkylation of Some Pyrimidine and Purine Derivatives Using Microwave-Assisted Methods. Heterocycles 2001, 55, 291-301.

14. http://www.chemie.uni-oldenburg.de/oc/metzger/eatos/

15. Eissen, M.; Metzger, J. O. Environmental Performance Metrics for Dayly Use in Synthetic Chemistry. Chem. Eur. J. 2002, 8, 3580-3585.

Sample Availability: Available from the authors.

(c) 2007 by MDPI (http://www.mdpi.org). Reproduction is permitted for noncommercial purposes. 